\title{
MAPPINGS OF TREES AND THE FIXED POINT PROPERTY
}

\author{
M. M. MARSH
}

\begin{abstract}
We investigate weakly confluent, universal, and related mappings of trees and their relationships to the fixed point property for tree-like continua. This investigation leads to some new results, to generalizations of some known results, and to a partial solution of a question of $\mathrm{H}$. Cook.
\end{abstract}

\section{INTRODUCTION}

In 1969, R. H. Bing [2] asked if each tree-like continuum has the fixed point property (f.p.p.). David Bellamy [1] answered Bing's question by giving an example of a tree-like continuum that admits a fixed-point-free mapping. Shortly thereafter, Oversteegen and Rogers [14] gave inverse limit descriptions of treelike continua without the f.p.p. Viewing tree-like continua as inverse limits of trees, one might add conditions to either the bonding mappings or the projection mappings in an effort to obtain fixed point theorems. We discuss results of this nature below.

W. Holsztynski called a mapping $f: X \rightarrow Y$ universal if for each mapping $g: X \rightarrow Y$, there is a point $x$ in $X$ such that $f(x)=g(x)$. Holsztynski [7, Corollary 1] proved the following theorem.

Theorem 1.1. If $X$ is an inverse limit of absolute neighborhood retracts with universal bonding mappings, then $X$ has the fixed point property.

In the proof of this theorem, Holsztynski showed that the projection mappings must also be universal. It is then easy to show that $X$ has the f.p.p.

In 1941, A. D. Wallace [16] showed that monotone maps from continua onto generalized trees are universal. In 1967, H. Schirmer [15] generalized Wallace's result by replacing monotone with weakly monotone. The next theorem follows from Schirmer's result.

Theorem 1.2. If $X$ is a tree-like continuum with weakly monotone projection mappings, then $X$ has the f.p.p.

Weakly monotone maps between finite trees must be confluent. C. A. Eberhart and J. B. Fugate [7] have shown that confluent maps of trees are weakly

Received by the editors November 13, 1987 and, in revised form, April 12, 1988.

1980 Mathematics Subject Classification (1985 Revision). Primary 54H25; Secondary 54F20.

Key words and phrases. Weakly confluent, universal, fixed point property, tree, inverse limit, tree-like, simple fold. 
arc preserving which, in turn, are universal. Theorem 1.3 below follows from their result and Theorem 1.1.

Theorem 1.3. If $X$ is an inverse limit of trees with weakly arc preserving bonding mappings, then $X$ has the f.p.p.

The author [8, Theorem 1] has shown that mappings between trees which have restrictions to $u$-mappings are universal. It was also shown in [8] that the class of such mappings properly contains the class of weakly arc preserving mappings. Hence, we have, in the following theorem, the most general known fixed point result for inverse limits of trees where conditions are placed only on the bonding mappings.

Theorem 1.4. If $X$ is an inverse limit of trees and each bonding mapping has a restriction that is a u-mapping, then $X$ has the f.p.p.

Other fixed point results for tree-like continua can be found in $[5,6,9-11$, and 12].

It is unknown if tree-like continua with weakly confluent (or even semiconfluent) bonding mappings must have the f.p.p. H. Cook has asked two questions pertaining to inverse limits with weakly confluent bonding or projection mappings.

(1) (Problem 122, U. of Houston Problem Book) Does each ( $n$-cell)-like continuum with weakly confluent projection mappings have the f.p.p.?

(2) (see [3]) Does each tree-like continuum with weakly confluent bonding mappings have the f.p.p.? The first of these two questions has been answered for $n=2$ by S. B. Nadler [13].

Theorem 1.5. If $X$ is disk (2-cell)-like with weakly confluent projection mappings, then $X$ has the f.p.p.

This paper is concerned with weakly confluent and related mappings of trees. We give a partial solution to Cook's second question which was obtained independently by the author in his dissertation [12] and by Eberhart and Fugate in [3]. Specifically, we show that inverse limits on a single tree with weakly confluent bonding maps must have the f.p.p. We also prove some interesting theorems about weakly confluent mappings between trees which may help solve Cook's second question. The theorems and corollaries of $\S 4$ establish cardinality comparisons between the endpoints, the branchpoints, and the edges emanating from branchpoints in the domain and image trees. In the case of finite trees, one of these theorems generalizes a result of Eberhart, Fugate, and Gordh [4, Lemma II.5]. Finally, in $\S 5$ we look at relationships between Cook's second question and S. Young's simple folds [17]. Note that semiconfluent (thus, weakly confluent) mappings between finite trees need not be universal and hence Theorem 1.1 cannot be applied directly to Cook's question. 


\section{Preliminary Definitions}

A continuum is a compact, connected metric space. A tree is a finite, connected, simply connected graph. Each continuous function will be referred to as a map or mapping. The topological space $X$ is said to have the fixed point property if for each mapping $f$ from $X$ to itself, there is a point $x$ in $X$ such that $f(x)=x$.

Suppose that $X$ is a tree. For $x \in X$, the order of $x$ in $X$, denoted $o_{X}(x)$, will be the cardinality of the set of components of $X-\{x\}$. We will omit the reference to $X$ if it is clear from the context. For any set $A$, the cardinality of $A$ will be denoted by $|A|$. We define the sets $E(X)$ and $B(X)$ of endpoints and branchpoints of $X$ respectively by

$$
E(X)=\left\{x \in X \mid o_{X}(x)=1\right\} \text { and } B(X)=\left\{x \in X \mid o_{X}(x) \geq 3\right\} .
$$

The set $V(X)=E(X) \cup B(X)$ will be referred to as the set of vertices of $X$.

For each pair of points $x_{1}, x_{2}$ in $X$, the unique arc in $X$ linearly ordered from $x_{1}$ to $x_{2}$ will be denoted by $\left[x_{1}, x_{2}\right] ;\left(x_{1}, x_{2}\right)$ and $\left(x_{1}, x_{2}\right]$ will denote respectively the open segment from $x_{1}$ to $x_{2}$, and the half-open segment from $x_{1}$ to $x_{2}$. The arc $\left[v_{1}, v_{2}\right]$ in $X$ will be called an edge of $X$ only in case $\left[v_{1}, v_{2}\right] \cap V(X)=\left\{v_{1}, v_{2}\right\}$. If $\left[v_{1}, v_{2}\right]$ is an edge of $X$ and one of $v_{1}$ or $v_{2}$ is in $E(X)$, then $\left[v_{1}, v_{2}\right]$ is a terminal edge of $X$. Otherwise, $\left[v_{1}, v_{2}\right]$ is an interior edge of $X$.

For each tree $X$ in this paper, we will assume that we have a metric $d$ defined on $X \times X$ so that each edge of $X$ has length one and, for $x_{1}, x_{2}$ in $X, d\left(x_{1}, x_{2}\right)$ is the length of the arc $\left[x_{1}, x_{2}\right]$.

\section{Combinatorial Lemmas}

Lemma 3.1. Suppose that $X$ is a tree with endpoint set $E$ and branchpoint set $B$. Then $|E|=2+\sum_{v \in B} o(v)-2|B|$.

Proof. Let $\alpha_{0}$ denote the number of vertices of $X$ and $\alpha_{1}$ the number of edges of $X$. It is well known that $\alpha_{0}-\alpha_{1}=1$. Since each edge of $X$ has exactly two vertices and, for $v \in V(X), o(v)$ is the number of edges emanating from $v, \sum_{v \in E \cup B} o(v)$ adds each edge twice; i.e., $2 \alpha_{1}=\sum_{v \in E \cup B} o(v)$. Thus,

$$
2 \alpha_{1}=\sum_{v \in E} o(v)+\sum_{v \in B} o(v)=|E|+\sum_{v \in B} o(v) .
$$

We have the following two expressions for $\alpha_{1}$ :

$$
\alpha_{1}=\alpha_{0}-1=|E|+|B|-1, \quad \alpha_{1}=\frac{1}{2}\left(|E|+\sum_{v \in B} o(v)\right) .
$$

Equating the two, we get that $|E|=2+\sum_{v \in B} o(v)-2|B|$.

Lemma 3.2. Suppose that $X$ and $Y$ are trees and $f: V(X) \rightarrow V(Y)$ is a function such that $f(B(X))$ is a proper subset of $B(Y), E(Y)$ is a subset of 
$f(E(X))$, and $\left.f\right|_{B(X)}$ is one-to-one. Then there is a point $v \in B(X)$ such that $o_{X}(v)>o_{Y}(f(v))$.

Proof. Suppose the contrary. Let $H=\{f(v) \mid v \in B(X)\}$ and $K=B(Y)-H$. By Lemma 3.1 and supposition, we get that

$$
\begin{aligned}
|E(X)| & =2+\sum_{v \in B(X)} o_{X}(v)-2|B(X)| \\
& \leq 2+\sum_{v \in B(X)} o_{Y}(f(v))-2|B(X)| .
\end{aligned}
$$

Since $\sum_{w \in K} o_{Y}(w)-2|K|>0$ and $\left.f\right|_{B(X)}$ is one-to-one, it follows that $2+\sum_{v \in B(X)} o_{Y}(f(v))-2|B(X)|<2+\sum_{w \in H} o_{Y}(w)-2|B(X)|+\sum_{w \in K} o_{Y}(w)-2|K|$

Thus,

$$
\begin{aligned}
|E(X)| & <2+\sum_{w \in H} o_{Y}(w)-2|B(X)|+\sum_{w \in K} o_{Y}(w)-2|K| \\
& =2+\sum_{w \in B(Y)} o_{Y}(w)-2|H|-2|K| \\
& =2+\sum_{w \in B(Y)} o_{Y}(w)-2|B(Y)| \\
& =|E(Y)| .
\end{aligned}
$$

However, $E(Y) \subseteq f(E(X))$ implies that $|E(Y)| \leq|E(X)|$, which is a contradiction.

Lemma 3.3. Suppose that $X$ and $Y$ are trees and $f: V(X) \rightarrow V(Y)$ is a function such that $f(B(X))=B(Y), E(Y) \subseteq f(E(X))$, and $\left.f\right|_{B(X)}$ is one-to-one. Then there is a point $v \in B(X)$ such that $o_{X}(v) \geq o_{Y}(f(v))$.

Proof. Suppose the contrary. By Lemma 3.1 and supposition,

$$
\begin{aligned}
|E(X)| & =2+\sum_{v \in B(X)} o_{X}(v)-2|B(X)| \\
& <2+\sum_{v \in B(X)} o_{Y}(f(v))-2|B(Y)| \\
& =|E(Y)| .
\end{aligned}
$$

As in Lemma 3.2, this yields a contradiction.

\section{WEAKLY CONFLUENT MAPS OF TREES}

A mapping $f: X \rightarrow Y$ is weakly confluent if for each subcontinuum $K$ of $Y$, there is a component $H$ of $f^{-1}(K)$ such that $f(H)=K$. Our first theorem shows that if $X$ and $Y$ are trees, the weak confluence of $f$ allows us to choose a continuum $H$ in $X$ such that $f$ maps $H$ onto $K$ in a certain manner. 
Theorem 4.1. Suppose that $f: X \rightarrow Y$ is a weakly confluent map from a tree $X$ onto a tree $Y$ and $K$ is a subtree of $Y$ such that $B(K)$ is not empty. Then there is a tree $H$ in $X$ such that

(1) $f(H)=K$,

(2) $B(H)$ is not empty,

(3) $E(K)=f(E(H))$, and

(4) either

a. $|B(H)| \geq|B(K)|$,

b. $\left.f\right|_{B(H)}$ is not one-to-one, or

c. there is a point $v \in B(H)$ such that $o_{H}(v)>o_{K}(f(v))$.

Proof. Let $\left\{K_{i}\right\}_{i=1}^{\infty}$ be a sequence of trees in $Y$ such that $\overline{\bigcup_{i=1}^{\infty} K_{i}}=K$, and for each integer $i \geq 1, K_{i} \subseteq K_{i+1} \subseteq K, E\left(K_{i+1}\right) \cap K_{i}$ is empty, and $B\left(K_{i}\right)=B(K)$. For each $i \geq 1$, let $M_{i}$ be a subtree of $X$ such that $f\left(M_{i}\right)=K_{i}$. Finally, for each $i \geq 1$, let $H_{i}$ be a subtree of $M_{i}$ which is minimal with respect to mapping onto $K_{i}$.

First we show that, for each $i \geq 1, f\left(E\left(H_{i}\right)\right) \subseteq E\left(K_{i}\right)$. Let $n$ be a positive integer, $e$ a point of $E\left(H_{n}\right)$, and suppose that $f(e)$ is in $K_{n}-E\left(K_{n}\right)$. Since $f^{-1}\left(E\left(K_{n}\right)\right)$ is closed and $e \notin f^{-1}\left(E\left(K_{n}\right)\right)$, we can pick a point $x$ in $H_{n}$ such that the arc $[x, e]$ does not intersect $f^{-1}\left(E\left(K_{n}\right)\right)$. Let $H_{n}^{\prime}=H_{n}-(x, e]$. We have that $H_{n}^{\prime}$ is a proper subtree of $H_{n}$ and $f\left(H_{n}^{\prime}\right)=K_{n}$, a contradiction.

We now show that $H_{i} \subseteq H_{j}-E\left(H_{j}\right)$ if $j>i \geq 1$ and $H_{i} \cap H_{j} \neq \varnothing$. Suppose there is a point $a$ in $E\left(H_{n}\right) \cap H_{m}$ for some $n>m \geq 1$. Then $f(a)$ is in $E\left(K_{n}\right) \cap K_{m}$; but, by our choice of the sequence $\left\{K_{i}\right\}_{i=1}^{\infty}$, this is a contradiction. Hence, if $j>i \geq 1$ and $H_{j}$ intersects $H_{i}$, then $H_{i} \subseteq H_{j}-E\left(H_{j}\right)$.

For some positive integer $j, \operatorname{diam}\left(K_{j}\right)>0$; and, for each $i \geq j, \operatorname{diam}\left(K_{i}\right)$ $\geq \operatorname{diam}\left(K_{j}\right)$. Thus, there does not exist an infinite subcollection of $\left\{H_{i}\right\}_{i=1}^{\infty}$ which is pairwise mutually exclusive. Therefore, we can choose a subsequence $\left\{H_{u(i)}\right\}_{i=1}^{\infty}$ of $\left\{H_{i}\right\}_{i=1}^{\infty}$ such that, for each $i \geq 1, H_{u(i)}$ intersects $H_{u(1)}$ and consequently, $H_{u(1)} \subseteq H_{u(i)}$. So, in fact, for each $i \geq 1, H_{u(i+1)}$ intersects $H_{u(i)}$, implying that $H_{u(i)}$ is a subset of $H_{u(i+1)}$. We have that $\left\{H_{u(i)}\right\}_{i=1}^{\infty}$ is monotonic increasing. We may assume, without loss of generality, that the sequence $\left\{H_{u(i)}\right\}_{i=1}^{\infty}$ is the sequence $\left\{H_{i}\right\}_{i=1}^{\infty}$.

Let $H=\bar{\bigcup}_{i=1}^{\infty} H_{i}$. We will show that $H$ satisfies properties 1-4. By the compactness of $X$ and $Y$ and the continuity of $f$, it follows that $f(H)=K$. This establishes 1 .

To prove property 2, we use an argument of Eberhart, Fugate, and Gordh [3, Theorem 2.1]. The proof is included here since we must make a couple of modifications.

Suppose that $B(H)$ is empty. Then $H$ is an arc, as is $H_{i}$, for each $i \geq$ 1. Since, for each $i \geq 1, E\left(H_{i+1}\right) \cap E\left(H_{i}\right)$ is empty, $H_{i+1}-H_{i}$ has exactly two components, $C_{i+1}$ and $D_{i+1}$. Now, $\lim _{i \rightarrow \infty}\left\{\operatorname{diam}\left(C_{i}\right)\right\}=0$, and $\lim _{i \rightarrow \infty}\left\{\operatorname{diam}\left(D_{i}\right)\right\}=0$. Let $\varepsilon$ be a positive number which is less than the 
length of each edge of $K$. Let $\delta$ be a positive number such that if $x_{1}$ and $x_{2}$ are in $X$ and $d\left(x_{1}, x_{2}\right)<\delta$, then $d\left(f\left(x_{1}\right), f\left(x_{2}\right)\right)<\varepsilon$. Since $B(K)$ is not empty, $|E(K)| \geq 3$. Let $a, b$, and $c$ be points of $E(K)$. For each $i \geq 1$, choose $a_{i}, b_{i}$, and $c_{i}$ in $E\left(K_{i}\right)$ such that $\lim _{i \rightarrow \infty}\left\{a_{i}\right\}=a$, $\lim _{i \rightarrow \infty}\left\{b_{i}\right\}=b$, and $\lim _{i \rightarrow \infty}\left\{c_{i}\right\}=c$. Since, for each $i \geq 1, f\left(H_{i}\right)=K_{i}$ and $E\left(K_{i+1}\right) \cap K_{i}$ is empty, there must be at least two of $f^{-1}\left(a_{i+1}\right), f^{-1}\left(b_{i+1}\right)$, and $f^{-1}\left(c_{i+1}\right)$ which intersect the same component of $H_{i+1}-H_{i}$. Let $n$ be a positive integer such that $\operatorname{diam}\left(C_{n}\right)<\delta, \operatorname{diam}\left(D_{n}\right)<\delta, d\left(a_{n}, a\right)<\varepsilon / 2$, $d\left(b_{n}, b\right)<\varepsilon / 2$, and $d\left(c_{n}, c\right)<\varepsilon / 2$. Suppose, without loss of generality, that $x_{1} \in C_{n} \cap f^{-1}\left(a_{n}\right)$ and $x_{2} \in C_{n} \cap f^{-1}\left(b_{n}\right)$. So, $d\left(x_{1}, x_{2}\right)<\delta$, which implies that $d\left(f\left(x_{1}\right), f\left(x_{2}\right)\right)=d\left(a_{n}, b_{n}\right)<\varepsilon$. Since $a$ and $b$ are in $E(K)$ and $\varepsilon$ was chosen less than the length of each edge of $K$, we get that $2 \varepsilon<d(a, b) \leq$ $d\left(a, a_{n}\right)+d\left(a_{n}, b_{n}\right)+d\left(b_{n}, b\right)$. Thus, $2 \varepsilon<\varepsilon / 2+d\left(a_{n}, b_{n}\right)+\varepsilon / 2$; yielding $\varepsilon<d\left(a_{n}, b_{n}\right)$, a contradiction. Hence, $B(H)$ is not empty. This completes the verification of property 2 .

It is easy to see that $E(H)=H-\bigcup_{i=1}^{\infty} H_{i}$ and $E(K)=K-\bigcup_{i=1}^{\infty} K_{i}$. With this observation, we are ready to establish property 3.

Suppose that $b \in H-\bigcup_{i=1}^{\infty} H_{i}$ and $f(b) \in K_{n}$ for some $n \geq 1$. Let $W$ be an open set in $Y$ containing $f(b)$ such that $W \cap K_{n+1}=W \cap K_{i}$ for each $i \geq n+1$. Let $U$ be an open set in $X$ containing $b$ such that $f(U) \subseteq W$. Choose an integer $j>n+1$ such that $E\left(H_{j}\right) \cap U$ is not empty. Finally, by picking a point $x \in E\left(H_{j}\right) \cap U$, we get that $f(x) \in E\left(K_{j}\right) \cap W$ and thus, $f(x) \in K_{j} \cap W=$ $K_{n+1} \cap W$. So we have that $f(x) \in E\left(K_{j}\right) \cap K_{n+1}$, which contradicts our choice of the $K_{i}$ 's. Hence, it must be the case that $f\left(H-\bigcup_{i=1}^{\infty} H_{i}\right) \subseteq f(H)-\bigcup_{i=1}^{\infty} K_{i}$. We are now able to establish the following set inclusion,

$$
f(E(H))=f\left(H-\bigcup_{i=1}^{\infty} H_{i}\right) \subseteq f(H)-\bigcup_{i=1}^{\infty} K_{i}=K-\bigcup_{i=1}^{\infty} K_{i}=E(K) .
$$

For the opposite inclusion, we let $e \in E(K)$ and suppose that there is a point $a \in \bigcup_{i=1}^{\infty} H_{i}$ such that $f(a)=e$. Let $n$ be a positive integer for which $a \in H_{n}$. Then $e$ belongs to $K_{n} \cap E(K)$, a contradiction. Thus, $E(K) \subseteq f(E(H))$. The two inclusions yield property 3 .

We need only prove property 4 in order to complete the proof of the theorem.

Suppose there is a point $v \in B(H)$ such that $f(v) \notin B(K)$. Then part c of property 4 is satisfied. Hence, we assume that $f(B(H)) \subseteq B(K)$. Suppose that parts a and b of property 4 are not satisfied; i.e., $|B(H)|<|B(K)|$ and $\left.f\right|_{B(H)}$ is one-to-one. We have already verified property 3. Therefore, by Lemma 3.2, there is a point $v \in B(H)$ such that $o_{H}(v)>o_{K}(f(v))$.

For trees $X$ and $Y$ and a weakly confluent mapping $f: X \rightarrow Y$, Eberhart, Fugate, and Gordh [4] have shown that the branchpoints of $Y$ must be covered by the branchpoints of $X$; i.e., $B(Y) \subseteq f(B(X))$. We point out that this result also follows from Theorem 4.1. To see this, let $w \in B(Y)$ and choose a decreasing sequence of subtrees $\left\{K_{i}\right\}_{i=1}^{\infty}$ such that $w$ is a branchpoint of each 
$K_{i}$ and $\bigcap_{i=1}^{\infty} K_{i}=\{w\}$. For each $i \geq 1$, there is a tree $H_{i}$ in $X$ such that $f$ maps $H_{i}$ onto $K_{i}$ in the manner of Theorem 4.1; in particular, $B\left(H_{i}\right)$ is not empty. Choose a tree $H$ in $X$ that is a sequential limit of some subsequence of $\left\{H_{i}\right\}_{i=1}^{\infty}$. It follows that $f(H)=\{w\}$ and $H \cap B(X)$ is not empty. So, the branchpoints of $Y$ are covered by the branchpoints of $X$.

Corollary 4.2. Suppose that in part a of property 4, $|B(H)|=|B(K)|$. Then either $\left.f\right|_{B(H)}$ is not one-to-one or there is a point $v \in B(H)$ such that $o_{H}(v) \geq$ $o_{K}(f(v))$.

Proof. Suppose that $f(B(H)) \subseteq B(K)$ and $\left.f\right|_{B(H)}$ is one-to-one. Then $f(B(H))=B(K)$; therefore, by Lemma 3.3, there is a point $v \in B(H)$ such that $o_{H}(v) \geq o_{K}(f(v))$.

Corollary 4.3. Suppose $f: X \rightarrow Y$ is a weakly confluent mapping from a tree $X$ onto a tree $Y$ such that $\left.f\right|_{B(X)}$ is one-to-one. Then, $o_{X}(v) \geq o_{Y}(f(v))$ for each point $v$ in $B(X)$.

Proof. We mentioned earlier that the branchpoints of $Y$ must be covered by the branchpoints of $X$; i.e., $B(Y) \subseteq f(B(X))$. Let $v$ be a branchpoint of $X$. If $f(v)$ is not in $B(Y)$, then clearly $o_{X}(v)>o_{Y}(f(v))$. Suppose that $f(v) \in B(Y)$. Let $K$ be a tree in $Y$ such that $K$ intersects each component of $Y-\{f(v)\}$ and $K \cap f(B(X))=\{f(v)\}$. By Theorem 4.1, there is a tree $H$ in $X$ such that $f(H)=K, f(E(H))=E(K)$, and $B(H)$ is not empty. Since $\left.f\right|_{B(X)}$ is one-to-one and $K \cap f(B(X))=\{f(v)\}$, it follows that $B(H)=\{v\}$. Hence,

$$
o_{Y}(f(v))=o_{K}(f(v))=|E(K)| \leq|E(H)|=o_{H}(v) \leq o_{X}(v) .
$$

Corollary 4.4. Suppose that $X$ is a tree and $f: X \rightarrow X$ is a weakly confluent map of $X$ onto itself. Then, $o(v)=o(f(v))$ for each $v \in B(X)$.

Proof. Again, we have that $B(X) \subseteq f(B(X))$. Therefore, $f$ must be one-toone on $B(X)$. Let $v \in B(X)$. Since $B(X)$ is finite and $\left.f\right|_{B(X)}$ is one-to-one, there is a positive integer $n$ such that $f^{n}(v)=v$. By Corollary 4.3, for each $u \in B(X), o(u) \geq o(f(u))$. Thus, $o(v) \geq o(f(v)) \geq o\left(f^{n}(v)\right)=o(v)$. Hence, $o(v)=o(f(v))$.

Before stating the next theorem, we need a few definitions. If $H$ is a subtree or a point of a tree $X$, we define $s t(H)$ to be the union of all edges of $X$ that intersect $H$. The arc $s$ is said to be a leg of $s t(H)$ provided that $s$ is the closure of some component of $s t(H)-H$. Notice that each leg of $s t(H)$ contains an endpoint of $s t(H)$.

Suppose $f: X \rightarrow Y$ is a mapping between trees, $w \in B(Y),\left\{t_{i}\right\}_{i=1}^{n}$ are the legs of $s t(w)$, and $[u, v]$ is an arc in $X$ so that $f(u)=w$, but $f([u, v]) \neq$ $\{w\}$. We will say that $[u, v]$ has an initial image under $f$ if there is an integer $j \in\{1, \ldots, n\}$ and a point $x \in[u, v]$ such that $f(x) \in t_{j}-\{w\}$ and, $f\left(x^{\prime}\right) \in t_{j}$ 
for every $x^{\prime} \in[u, x]$. In this case, we will also say that $t_{j}$ is the initial image of $[u, v]$ under $f$. The reference to $f$ will be omitted if such reference is clear.

The next theorem generalizes, in the case of finite trees, Lemma II.5 of Eberhart, Fugate, and Gordh [4].

Theorem 4.5. Suppose that $f: X \rightarrow Y$ is a weakly confluent map of a tree $X$ onto a tree $Y, w \in B(Y)$, and $s t(w)$ has legs $\left\{t_{i}\right\}_{i=1}^{n}$. Let $\left\{M_{i}\right\}_{i=1}^{m}$ be the collection of components of $f^{-1}(w)$ that contain a branchpoint. Suppose also that, for each $i \in\{1, \ldots, m\}$ and each leg $s$ of $s t\left(M_{i}\right), s$ has an initial image. Then there is an integer $i \in\{1, \ldots, m\}$ such that for each $j \in\{1, \ldots, n\}$, there is a leg $s$ of $s t\left(M_{i}\right)$ whose initial image is $t_{j}$.

Proof. For each integer $i \in\{1, \ldots, m\}$, let $\left\{M_{r}(i)\right\}_{r=1}^{\infty}$ be a decreasing sequence of trees in $X$ such that $\bigcap_{r=1}^{\infty} M_{r}(i)=M_{i}$, and, for each $r \geq 1$,

(i) $M_{r}(i)$ intersects each component of $X-M_{i}$,

(ii) $M_{r}(i) \cap B(X)=M_{i} \cap B(X)$,

(iii) $\left(E\left(M_{r}(i)\right)-E(X)\right) \cap f^{-1}(w)$ is empty, and

(iv) $\left(E\left(M_{r}(i)\right)-E(X)\right) \cap M_{r+1}(i)$ is empty.

In addition, for each $i \in\{1, \ldots, m\}$, let $k_{i}$ be a positive integer such that if $r \geq k_{i}$ and if $s$ is the closure of a component of $M_{r}(i)-M_{i}$, then $f(s)$ meets at most one component of $Y-\{w\}$. Such an integer exists since each leg of $\operatorname{st}\left(M_{i}\right)$ has an initial image.

Now let $K$ be a tree in $Y$ such that $K$ intersects each component of $Y-$ $\{w\}, K \cap B(Y)=\{w\}, K \cap f(B(X))=\{w\}$, and $K \cap f\left(E\left(M_{k_{i}}(i)\right)-E(X)\right)$ is empty for each $i \in\{1, \ldots, m\}$. Also choose $K$ so that for each interior edge $\left[v_{1}, v_{2}\right]$ of $X$ with the property that $f\left(v_{1}\right)=w, f\left(v_{2}\right)=w$, but $f\left(\left[v_{1}, v_{2}\right]\right) \neq$ $\{w\}, f\left(\left[v_{1}, v_{2}\right]\right)$ intersects the complement of $K$. Applying Theorem 4.1, we choose a tree $H$ in $X$ such that properties 1-4 hold. By choice of $K$, if $v \in B(X) \cap H$, then $f(v)=w$. Suppose that $v_{1}$ and $v_{2}$ are in $B(X) \cap H$, and that $f\left(\left[v_{1}, v_{2}\right]\right) \neq\{w\}$. Now, $f\left(v_{1}\right)=w=f\left(v_{2}\right)$, and thus, $f\left(\left[v_{1}, v_{2}\right]\right)$ must intersect the complement of $K$; but, $\left[v_{1}, v_{2}\right] \subseteq H$, which yields a contradiction. Hence, for each pair of points $v_{1}$ and $v_{2}$ in $B(X) \cap H, f\left(\left[v_{1}, v_{2}\right]\right)=\{w\}$. Let $M$ be the unique minimal tree (perhaps degenerate) in $H$ such that $B(X) \cap$ $H \subseteq M$. Then $f(M)=\{w\}$. Let $i$ be the integer in $\{1, \ldots, m\}$ for which $M \subseteq M_{i}$.

We are now ready to show that the conclusion holds for the integer $i$ chosen above. Let $t_{j}$ be a leg of $s t(w)$. Let $[w, b]$ be the terminal edge of $K$ such that $[w, b] \subseteq t_{j}$. Choose a point $a \in E(H)$ for which $f(a)=b$. Finally, let $v$ be the nearest point of $B(X) \cap H$ to $a$. Then $v \in M_{i}$ and $[v, a]$ is a subset of some terminal edge $s$ of $s t\left(M_{i}\right)$. Suppose that $f([v, a]) \nsubseteq[w, b]$. Since $[v, a] \subseteq H$, it follows that $f([v, a]) \subseteq K$ and $f([v, a])$ must intersect two components of $Y-\{w\}$. Hence, by our choice of $k_{i},[v, a] \nsubseteq M_{k_{i}}(i)$. Let $e \in[v, a] \cap E\left(M_{k_{i}}(i)\right)$ and let $[w, c]$ be the terminal edge of $K$ such that $f([v, e]) \subseteq[w, c]$. Now, $e \notin E(X)$. So, we have that $f(e) \in K$ and $f(e) \in$ 
$f\left(E\left(M_{k_{i}}(i)\right)-E(X)\right)$, which contradicts our choice of $K$. Thus, $f([v, a]) \subseteq$ $[w, b]$. Therefore, $t_{j}$ is the initial image of the leg of $\operatorname{st}\left(M_{i}\right)$ which is contained in $s$.

We point out that the assumption

(*) "for each $i=1, \ldots, m$ and each leg $s$ of $s t\left(M_{i}\right), s$ has an initial image"

cannot be eliminated from the hypothesis of Theorem 4.5. Also, we cannot generalize this result even to a branchpoint covering theorem by assuming that $Y$ is a finite tree and $X$ is a fan (see Ex. III.1 of [4]). We do, however, have the following corollaries.

Corollary 4.6. Property $(*)$ may be replaced by the assumption that $f^{-1}(w)$ has finitely many components.

Corollary 4.7. Property (*) may be replaced by the assumption that $f$ is piecewise linear.

Next we wish to show that if $f$ is a weakly confluent mapping of a tree onto itself, then $f$ is universal. It is easy to construct examples of weakly confluent (even semiconfluent) mappings between different trees that are not universal.

Lemma 4.8. If $f: T \rightarrow T$ is a weakly confluent map from a tree onto itself and $\left[w_{1}, w_{2}\right]$ is an edge of $T$ with $w_{1} \in B(T)$, then there is a unique branchpoint $v_{1}$ in $T$ and an arc $\left[v_{1}, v_{2}\right]$ such that $f\left(v_{1}\right)=w_{1}, f\left(v_{2}\right)=w_{2}$, and $f\left(\left[v_{1}, v_{2}\right]\right)=$ $\left[w_{1}, w_{2}\right]$. Moreover, if $w_{2} \in B(T)$, then $v_{2}$ may be chosen from $B(T)$.

Proof. By Theorem 4.1 (or [4]) and the finiteness of $B(T)$, it follows that for each branchpoint $w$ of $T$, there is exactly one branchpoint $w^{\prime}$ of $T$ such that $f\left(w^{\prime}\right)=w$. Thus, there is a one-to-one correspondence induced by $f$ on the branchpoints of $T$. Also, by Corollary 4.4,o(w)=o( $\left.w^{\prime}\right)$ for each $w \in B(T)$.

Let $v_{1}=w_{1}^{\prime}$. Assume that $w_{2} \in E(T)$. For each open connected set $U$ containing $w_{1}$, there is a tree $H_{U}$ as in Theorem 4.1 such that $f\left(H_{U}\right)=\bar{U}$ and $B\left(H_{U}\right)$ is not empty. It follows that there is an arc $\left[v_{1}, v_{2}\right]$ such that $f\left(v_{2}\right)=w_{2}$ and $f\left(\left[v_{1}, v_{2}\right]\right)=\left[w_{1}, w_{2}\right]$.

We now suppose that $w_{2} \in B(T)$. Let $v_{2}=w_{2}^{\prime}$. It is clear that $\left[w_{1}, w_{2}\right] \subseteq$ $f\left(\left[v_{1}, v_{2}\right]\right)$. Suppose that $f\left(\left[v_{1}, v_{2}\right]\right) \nsubseteq\left[w_{1}, w_{2}\right]$. We assume without loss of generality that $f\left(\left[v_{1}, v_{2}\right]\right)$ intersects some component of $T-\left[w_{1}, w_{2}\right]$ which has $w_{1}$ in its closure. Let $x_{1}$ and $x_{2}$ be points in $\left[v_{1}, v_{2}\right]$ such that $f\left(x_{1}\right) \neq$ $f\left(x_{2}\right), f\left(x_{1}\right) \notin B(T), w_{1}$ separates $f\left(x_{1}\right)$ from $w_{2}$, and $f\left(x_{1}\right)$ separates $f\left(x_{2}\right)$ from $w_{1}$. Let $K$ be the closure of the component of $T-\left\{f\left(x_{1}\right)\right\}$ that contains $w_{1}$. So, $K$ is a tree which contains the points $w_{1}, w_{2}$, and $f\left(x_{1}\right)$. Choose a subtree $H$ of $T$ as in Theorem 4.1. Now, $\left.f\right|_{B(H)}$ is one-to-one, and $o_{H}(v) \leq o_{T}(v)=o_{T}(f(v))=o_{K}(f(v))$ for each $v \in B(H)$. Thus, by property 4 of Theorem 4.1, we must have that $|B(H)| \geq|B(K)|$. Since $B(K)=B(T) \cap K$ and $\left.f\right|_{B(H)}$ is one-to-one, it follows that $v_{1}$ and $v_{2}$ are in $H$. But $f\left(x_{2}\right) \notin K$. So $x_{2} \notin H$, a contradiction. 
Theorem 4.9. If $f: T \rightarrow T$ is a weakly confluent map of a tree onto itself, then $f$ is universal.

Proof. Assume that $g: T \rightarrow T$ is a mapping and $f(x) \neq g(x)$ for $x \in T$. For each branchpoint $u$ of $T$, let $u^{\prime}$ be the unique branchpoint of $T$ such that $f\left(u^{\prime}\right)=u$ and let $\hat{g}(u)=g\left(u^{\prime}\right)$. So, for each $u \in B(T), \hat{g}(u) \neq u$. It follows that (see Lemma in [11]) there exists neighboring vertices $v$ and $w$ in $T$ such that $\hat{g}(v)=g\left(v^{\prime}\right)$ is in the component of $T-\{v\}$ that contains $w$ and $\hat{g}(w)=g\left(w^{\prime}\right)$ is in the component of $T-\{w\}$ that contains $v$. By Lemma 4.8, $f\left(\left[v^{\prime}, w^{\prime}\right]\right)=[v, w]$. It follows that $\left.g\right|_{\left[v^{\prime}, w^{\prime}\right]}$ and $\left.f\right|_{\left[v^{\prime}, w^{\prime}\right]}$ have a coincidence point, which contradicts our assumption.

The following fixed point theorem follows from Theorems 4.9 and 1.1.

Theorem 4.10. Suppose that $T$ is a tree and $X=\lim \left\{X_{i}, g_{i}^{i+1}\right\}$, where, for each $i \geq 1, X_{i}=T$ and $g_{i}^{i+1}$ is a weakly confluent mapping of $X_{i+1}$ onto $X_{i}$. Then $X$ has the fuxed point property.

\section{WEAKLY CONFLUENT SIMPLE FOLDS}

In [17], $\mathrm{S}$. Young introduces the following definitions. Let $T_{1}$ and $T_{2}$ be trees, $p \in T_{1}$, and $T_{a}$ and $T_{b}$ subtrees of $T_{1}$ with $T_{a} \cup T_{b}=T_{1}$ and $T_{a} \cap T_{b}=$ $\{p\}$.

If $\beta: T_{1} \rightarrow T_{2}$ is not a homeomorphism but each restriction $\left.\beta\right|_{T_{a}}$ and $\left.\beta\right|_{T_{b}}$ is a homeomorphism, then $\beta$ is called a fold. If one of the trees $T_{a}$ or $T_{b}$ is the closure of a component of $T_{1}-\{p\}$, then $\beta$ is called a simple fold.

In Theorem 1 of [17], Young gives a procedure for factoring light maps of trees into simple folds; he later proves the following result [17, Corollary 2].

Theorem 5.1. If $X$ is a tree-like continuum, then $X$ is the limit of an inverse system $T_{1} \leftarrow T_{2} \leftarrow \cdots \leftarrow X$, where each of the bonding maps is a simple fold.

With Cook's question in mind, it is natural to ask the following two questions.

(i) Do light weakly confluent maps between trees factor (using Young's technique) into weakly confluent simple folds?

(ii) Are weakly confluent simple folds universal?

An affirmative answer to both of these questions would give an affirmative answer to Cook's question (recall Theorem 1.1). Corollary 5.3 below gives an affirmative answer to question (ii). In regard to question (i), suppose that we have a weakly confluent map $f: T_{1} \rightarrow T_{2}$ between trees that factors into a finite sequence of weakly confluent simple folds. It follows from Theorem 5.2 below that some restriction of $f$ must be a homeomorphism with image $T_{2}$. Hence, the only weakly confluent maps of trees that we can hope to factor into weakly confluent simple folds are those that have restrictions which are homeomorphisms onto the image tree. It is well known that such mappings are universal. Hence, this approach to answering Cook's question yields only a well-known special case. 
Theorem 5.2. Suppose that $f: T_{1} \rightarrow T_{2}$ is a weakly confluent simple fold. Then some restriction of $f$ is a homeomorphism onto $T_{2}$.

Proof. Let $p, T_{a}$, and $T_{b}$ be given as in the definition of a simple fold. Suppose that $T_{a}$ is the closure of a component of $T_{1}-\{p\}$. By Obs. (3) in [17], on some open set $U$ with $p \in U, f$ identifies exactly two components of $\left(T_{1} \cap U\right)-\{p\}$. Let $H_{1}$ and $H_{2}$ be these components and let $K=f\left(H_{1}\right)=f\left(H_{2}\right)$. Now, one of $H_{1}$ or $H_{2}$ is a subset of $T_{a}$; for otherwise, $H_{1} \cup H_{2} \subseteq T_{b}$ implying that $\left.f\right|_{T_{b}}$ is not a homeomorphism. Assume that $H_{1} \subseteq T_{a}$. Similarly, it follows that $H_{2} \subseteq T_{b}$. Since $\left.f\right|_{T_{a}}$ and $\left.f\right|_{T_{b}}$ are homeomorphisms and $f$ is weakly confluent, one of $C_{1}$ or $C_{2}$ maps onto $D$, where $C_{i}(i=1,2)$ is the component of $T_{1}-\{p\}$ containing $H_{i}$ and $D$ is the component of $T_{2}-\{f(p)\}$ containing $K$. Say $f\left(C_{k}\right)=D$, where $k$ is either 1 or 2 . Let $R=T_{b}-C_{2}$. Again, from the weak confluence of $f$, it follows that $f(R)=T_{2}-D$. But now we have that $f\left(C_{k}\right)=D, f(R)=T_{2}-D$, and each of $\left.f\right|_{C_{k}}$ and $\left.f\right|_{R}$ is a homeomorphism (notice that $C_{k}$ is either a subset of $T_{a}$ or of $T_{b}$, and $R$ is a subset of $T_{b}$ ). It follows that $\left.f\right|_{C_{k} \cup R}$ is a homeomorphism with image $T_{2}$.

Corollary 5.3. If $f: T_{1} \rightarrow T_{2}$ is a weakly confluent simple fold, then $f$ is universal.

Recall that the proof of Theorem 1.1 shows that if the bonding mappings are universal, then so are the projection mappings. Actually, one only needs that the projection mappings are weakly universal to show that the inverse limit has the fixed point property. A mapping $f: X \rightarrow Y$ is weakly universal if for each mapping $g: X \rightarrow X$, there is a point $x \in X$ such that $f(x)=f g(x)$. In this regard, we ask the following question.

Question 5.4. If $X$ is an inverse limit of trees with weakly confluent bonding mappings, must the projection mappings be weakly universal?

Other questions pertaining to universal, weakly universal, and related mappings can be found in the Sacramento State Topology Conference Problem Session Notes (April 1987).

The author gratefully acknowledges the help he received from I. J. Christopher in learning to use the TEX technical typesetting program and from C. L. Hagopian in revising and improving this paper.

\section{REFERENCES}

1. D. Bellamy, A tree-like continuum without the fixed point property, Houston J. Math. 6 (1979), $1-14$.

2. R. H. Bing, The elusive fixed point property, Amer. Math. Monthly 76 (1969), 119-132.

3. C. A. Eberhart and J. B. Fugate, Weakly confluent maps on trees, General Topology and Modern Analysis, Academic Press, New York, 1981, pp. 209-215.

4. C. A. Eberhart, J. B. Fugate, and G. R. Gordh, Jr., Branchpoint covering theorems for confluent and weakly confluent maps, Proc. Amer. Math. Soc. 55 (1976), 409-415. 
5. C. L. Hagopian, Fixed points of tree-like continua, Contemp. Math., vol. 78, Providence, R.I., 1988, pp. 131-137.

6. $\ldots$, Fixed points of arc-component-preserving maps, Trans. Amer. Math. Soc. 306 (1988), 411-420.

7. W. Holsztysnki, "Universal mappings and fixed point theorems", Bull. Polon. Acad. Sci. 15 (1967), 433-438.

8. M. M. Marsch, u-mappings on trees, Pacific J. Math. 127 (1987), 373-387.

9. $\ldots$, A fuxed point theorem for inverse limits of fans, Proc. Amer. Math. Soc. 91 (1984), 139-142.

10. $\ldots$, A fixed point theorem for inverse limits of simple n-ods, Topology Appl. 24 (1986), 213-216.

11. __ E-mappings onto a tree and the fixed point property, preprint.

12. __ Fixed point theorems for certain tree-like continua, Dissertation, Univ. of Houston, 1981.

13. S. B. Nadler, Jr., Universal mappings and weakly confluent mappings, Fund. Math. 110 (1980), 221-235.

14. L. G. Oversteegen and J. T. Rogers, Jr., Fixed-point-free maps on tree-like continua, Topology Appl. 13 (1982), 85-95.

15. H. Schirmer, Coincidence producing maps onto trees, Canad. Math. Bull. 10 (1967), 417-423.

16. A. D. Wallace, A fixed point theorem for trees, Bull. Amer. Math. Soc. 47 (1941), 757-760.

17. S. W. Young, Tree-like continua and simple bonding maps, Topology Proc. 7 (1982), 181-190.

Department of Mathematics, California State University, Sacramento, Sacramento, CALIFORNIA 95819-2694 